\title{
ENHANCED CORTICOMOTOR EXCITABILITY WITH DYNAMIC FATIGUING EXERCISE OF THE LOWER LIMB IN MULTIPLE SCLEROSIS
}

Gary W Thickbroom, Paul Sacco, Deborah L Faulkner, Allan G Kermode, Frank L Mastaglia

Centre for Neuromuscular and Neurological Disorders, University of Western Australia, Queen Elizabeth II Medical Centre, Perth, Western Australia WA 6009

Address for Correspondence:

G W Thickbroom

Centre for Neuromuscular and Neurological Disorders, M518

University of Western Australia

Queen Elizabeth II Medical Centre

Nedlands, WA 6009 Australia.

Email: gthickbr@cyllene.uwa.edu.au

Tel: +61 893474479

Fax +61 893463487

Acknowledgement: This study was generously supported by the Trish Multiple Sclerosis Research Foundation.

Keywords: Multiple Sclerosis, fatigue, dynamic exercise, TMS 


\begin{abstract}
Objective: To determine if task performance and fatigability during rapid repetitive exercise of the lower limb differ in a group of MS subjects compared to a control group, and what central changes accompany the development of fatigue and the period of recovery.

Methods: Transcranial magnetic stimulation (TMS) was used to compare motor evoked potential (MEP) responses between 10 clinically definite MS subjects (7F, 33-64 years of age; EDSS $\leq 4$; MRC grade $\geq 4 / 5$ ) and 13 control subjects during and after 5 bouts of a 15 -second maximum rate foot-tapping task performed at 1 minute intervals.
\end{abstract}

Results: Maximum voluntary contraction (MVC) force of ankle dorsiflexion was lower (15\%) in the MS group compared to controls, however there were no differences in the rate of foot tapping. The rate of foot tapping decreased during each bout of exercise to a comparable degree in both groups, but there was no overall deterioration in performance across the 5 repeat bouts in either group. MS subjects showed a greater decline in strength than controls after exercise (20.7 $\pm 7.7 \%$ vs. $6 \pm 3.6 \%$; $\mathrm{p}<0.05$ ). MEP amplitude increased significantly for the exercised limb in both groups, but the increase was greater in MS subjects (65.9 $\pm 27 \%$ vs. $31 \pm 19.6 \%$; MS vs. control; $\mathrm{p}<0.05)$. MEP amplitude also increased for the non-exercised limb in controls (40.6 $\pm 15.6 \%, \mathrm{p}<0.01)$ but not in MS subjects.

Conclusions: Mild to moderately affected MS subjects can perform a fatiguing exercise requiring a high level of central motor control but this is associated with a greater strength loss and increase in corticomotor excitability compared to unaffected individuals.

Significance: Central adaptive processes are likely to have a significant role in maintaining task performance in MS. 


\section{INTRODUCTION}

Fatigue is a common and disabling symptom in patients with multiple sclerosis (MS) that interferes with the performance of various everyday activities, such as walking, and reduces quality of life. The pathophysiological basis for the fatigue that develops during such activities is still poorly understood. It has been assumed that fatigue is related to impaired conduction in demyelinated central motor pathways. In keeping with this premise slowing of central motor conduction has been reported during fatiguing exercise in some transcranial magnetic stimulation (TMS) studies [9]. However, in a study using paired-pulse stimulation no evidence of frequency-dependent conduction block was found [14]. Other TMS studies have shown that MS subjects have reduced resting motor evoked potential (MEP) amplitudes at rest, but are able to increase corticomotor excitability and central motor drive to a greater degree than normal during sustained or repetitive activities $[7,16]$.

Most previous studies in MS subjects have employed fatiguing protocols for the upper limb or hand and few studies have attempted to investigate the neurophysiological basis for fatigue in the lower limbs. Lower limb fatigue can be important for functional activities such as walking, and the lower limbs may have an greater susceptibility to central fatigue because of the longer corticospinal pathway. Using an intermittent incremental isometric contraction protocol to induce fatigue in the ankle dorsiflexors, and tetanic supramaximal stimulation of the peroneal nerve, Kent-Braun et al. [5] found that central activation was submaximal in some MS subjects when fatigue developed, but concluded that this was not the sole cause of fatigue. There have been few TMS studies during fatiguing lower limb exercise. Schubert et al. [13] recorded MEP responses from the tibialis anterior muscle after a walking task and found a greater degree of 
MEP depression than normal in MS subjects, but there was a marked variability in responses in individual subjects

In the present study we investigated the changes in motor performance and in the excitability of the corticomotor projection to the lower limb during a repetitive exercise protocol of the ankle dorsiflexors in a group of MS subjects. The aims of the study were to determine whether the MEP changes associated with this exercise protocol are similar to those reported in upper limb muscles and if these changes are correlated with alterations in motor performance. We also wished to determine whether, in addition to increased force loss during this type of activity, there is deterioration in other movement parameters such as repetition rate which could be related to fatigue of central motor control mechanisms.

\section{METHODS}

\section{Subjects}

Ten subjects (7 female, 33-64 years of age) with early to moderately advanced clinically definite relapsing-remitting MS according to the revised McDonald criteria [10] (Expanded Disability Status Scores $($ EDSS $) \leq 4$; lower limb MRC grades $\geq 4 / 5)$ and 13 normal healthy controls (9 female; 21-57 years of age) participated in the study, with approval from the Committee of Human Rights of the University of Western Australia.

MS subject details are presented in Table 1. Details of all medications were recorded. Individuals were excluded from the study if they had clinical depression; severe pyramidal weakness (MRC grade < 4) or incoordination; sensory deficits on one or both sides; and/or, used psychotropic or anticonvulsant medications. Subjects on $\beta$-interferon were studied on the day prior to injection. Lower limb function was additionally assessed by a 50m timed walk. 


\section{Exercise Task}

Subjects were seated and both feet positioned on a raised platform. A marker was placed above the dominant foot at a height that corresponded to a comfortable range of ankle dorsiflexion. Subjects were asked to tap their dominant foot between the platform and the level of this marker keeping the heel on the platform. Subjects performed this exercise task as fast as possible for 15 seconds, followed by 45 seconds of rest during which TMS recordings were performed. This combined 1-minute exercise-rest period was repeated 5 times consecutively.

\section{Force and task-performance measures}

Maximum voluntary contraction (MVC) force of the ankle dorsiflexors was measured isometrically in mid-range using a dynamometer (Penny and Giles) attached to a support positioned over the foot. The number of taps and inter-tap interval was determined using an accelerometer, attached to the foot, which recorded a spike with each tap of the platform. MVC force was measured at baseline and immediately after the last bout of exercise.

\section{Electromyography (EMG)}

EMG was recorded from electrodes of $5 \mathrm{~mm}$ diameter placed over the tibialis anterior (TA) muscle on both sides. The active electrode was placed over the belly of the TA muscle and the reference electrode 4cm proximal to this site. The signals were amplified by 1000 and were pass-filtered above $2 \mathrm{~Hz}$ and below $2 \mathrm{kHz}$.

\section{Transcranial Magnetic Stimulation (TMS)}

A Magstim 200 magnetic stimulator with a double-cone coil (9cm inner diameter) was used to elicit motor evoked potential (MEP) responses in the TA at rest. The coil was held over the 
midline, so that stimulation elicited MEPs on both sides. The position along the antero-posterior axis giving the largest MEPs was determined by initial exploration. TMS intensity was set to $10 \%$ of stimulator output above the intensity at which a MEP could be elicited with 3 out of 4 stimuli. Baseline MEPs were measured in groups of 4 stimuli $(0.2 \mathrm{~Hz})$ each minute for 5 minutes prior to exercise. Five stimuli were delivered at 5 second intervals starting 10 seconds after each exercise bout, and continuing at 1 minute intervals for 5 minutes after the final bout.

\section{Data Analysis}

For each of the five 15-second bouts of exercise, tap rate $(\mathrm{Hz})$ was calculated for the first and last 5 seconds of exercise. Trends with time were investigated by normalising tap rate to the first bout, and trends across bouts tested with Pearson's product-moment correlation. MVC force at baseline and after the exercise protocol was compared using Student’s t-test.

Peak-peak MEP amplitude was used as a measure of corticomotor excitability. Additional methods for quantitating the MEP were investigated, including area under the curve and polyphasic measures (turns analysis), however these approaches did not provide additional information. Mean MEP amplitude was determined for each inter-bout interval, giving 4 measures associated with the exercise protocol. The MEP data set obtained after the last bout of exercise was treated as the first measurement of the recovery period. MEP data was normalised to baseline, and comparisons were made by ANOVA (group x time) and pooled MEP data (separately for inter-bout and recovery periods) tested for difference from baseline (100\%) by ztest. All results are mean \pm standard error of the mean (SEM). 


\section{RESULTS}

\section{Strength}

At baseline, MVC force during ankle dorsiflexion was 15\% lower for the MS group compared to controls ( $137 \pm 10 \mathrm{~N}$ vs. $162 \pm 8 \mathrm{~N}$; MS vs. controls respectively; $\mathrm{p}<0.05)$. After all 5 bouts of exercise, MVC force was significantly reduced in both groups, to $111 \pm 15 \mathrm{~N}$ for the MS group ( $<<0.02)$, and $150 \pm 7 \mathrm{~N}$ for the control group $(\mathrm{p}<0.05)$. The mean percentage reduction in force was greater for the MS group than controls (20.7 $\pm 7.7 \%$ vs. $6 \pm 3.6 \%$; $<0.05)$.

\section{Tapping}

During the first 5 seconds of the first bout of exercise, MS subjects and controls tapped at comparable rates $(3.6 \pm 0.4 \mathrm{~Hz}$ vs. $3.9 \pm 0.3 \mathrm{~Hz}$ respectively; $\mathrm{p}>0.05)$. For the first 5 seconds of each subsequent bout of exercise, tap rate (normalised to the first bout) increased linearly for both groups (Figure 1; Pearson's $\mathrm{r}^{2}=0.9$ (MS and control); $\mathrm{p}<0.05$ ).

For each bout, tap rate decreased during performance of the task. During the last 5 seconds of the first bout of exercise, tap rate was significantly reduced (compared to the first 5 seconds) in MS and controls, and by a similar amount ( $\sim 8 \%$ ) for both groups (MS: $3.3 \pm 0.3 \mathrm{~Hz}, \mathrm{p}<0.05$; Control: 3.6 $\pm 0.2 \mathrm{~Hz}, \mathrm{p}<0.05)$. However, there was no significant change in normalised tape rate for the last 5 seconds of each subsequent bout for either group (Figure 1).

MEP

MEP latency was delayed in the MS group relative to controls for both limbs (right TA: 37.1 $\pm 4.4 \mathrm{~ms}$ vs. $29.0 \pm 0.5 \mathrm{~ms}$; left TA: $37.2 \pm 3.7$ vs. $28.8 \pm 0.5$; MS vs. controls; $\mathrm{p}<0.05$ ). There was 
no significant difference in MEP amplitude between groups for either limb $(0.75 \pm 0.23 \mathrm{mV}$ vs. $1.14 \pm 0.12 \mathrm{mV}$; MS vs. control; $\mathrm{p}>0.05)$.

In the controls, between exercise bouts, MEP amplitude was raised above baseline for both the exercised and non-exercised limbs (Figure 2B; 31 $\pm 19.6 \%$, $\mathrm{p}<0.05 ; 40.6 \pm 15.6 \%, \mathrm{p}<0.01$; exercised and non-exercised respectively; percentage increase from baseline; mean across interbout recordings). However in the MS group, MEP amplitude was increased for the exercised limb only (Figure 2A; 65.9 $\pm 27 \%, \mathrm{p}<0.01$ ). The percentage increase in MEP amplitude for the exercised limb was greater in the MS subjects compared to controls (65.9 $\pm 27 \%$ vs. $31 \pm 19.6 \%$; $\mathrm{p}<0.05)$

After exercise, MEP amplitude was not significantly different to baseline for either limb in either the MS or control groups (Figure 2).

\section{DISCUSSION}

Previous studies that have examined corticomotor changes associated with fatigue in MS have used sustained maximum voluntary contractions or repeated sub-maximal contractions with periods of rest. Perretti et al. [8] showed a greater post exercise facilitation (PEF - increased MEP amplitude in periods of rest during an intermittent exercise task) in MS than controls, and Nielsen and Norgaard [7] found an increase in PEF in MS subjects following sustained (non-fatiguing) contractions. In our previous study of intermittent submaximal fatiguing exercise of the hand, we also showed that corticomotor excitability was raised in MS relative to controls [16]. In the present study, we used a fatiguing task that requires a demanding level of motor control, rather than one that requires maximising motor drive, in order to further explore central mechanisms of fatigue in 
MS. The findings support the observations in the upper limb, and show that MEP amplitude is increased in association with a fatiguing foot-tapping task and that there is a greater increase in MEP amplitude in MS compared to control subjects. Thus both for upper and lower limbs and for isometric and dynamic fatiguing exercises, it appears that a similar central response to fatigue is occurring in both groups but that this is more pronounced in MS. The precise relationship between MEP amplitude and the development of fatigue is not certain, but an increase in MEP amplitude may reflect a change in the balance of intracortical excitation and inhibition that could underlie central adaptive processes directed at maintaining task performance as fatigue develops. We have also previously shown that there are changes in the corticomotor map of the hand in MS which are correlated with clinical and motor measures [15], further highlighting the role of neural plasticity in compensating for the effects of demyelination in MS.

MS and control subjects were able to maintain task performance across bouts, but tap-rate declined within each of the exercise periods. Thus each 45 second inter-bout resting period was sufficient to restore performance, and tap rate at the outset of each bout of exercise actually increased across bouts. One possibility for this increase is that subjects performed smaller foot movements. We were not able to record the magnitude of foot movement for each tap, however the taps were visually-guided between markers, and performance was closely monitored by an observer who provided verbal feedback and encouragement. Another possibility is that there may have been a practice effect across bouts. Foot tapping at maximal voluntary rate involves a high level of motor control, and it is likely that there would be some improvement in performance across trials as subjects became more practiced at the task.

While MS and control subjects tapped at comparable rates, the MS subjects were weaker according to isometric testing of MVC force during ankle dorsiflexion. This suggests that in MS 
tap-rate does not depend entirely on force generating ability, and that central control mechanisms that modulate rapid switching between agonist/antagonist muscle activation are important in regulating tap-rate. As well, the degree of force loss after the overall exercise protocol was substantially greater for the MS group than for controls, however there remained no difference in tap rate between groups. MS subjects also had delayed conduction time which could affect the timing of agonist and antagonist activation needed for fast tapping. That task performance was normal in the MS group in the presence of force loss and conduction delay suggests the presence of central adaptive processes that can act to compensate for these factors and maintain task performance. Such processes are almost certainly present in healthy controls, as for example there is no significant loss of motor skill after fatiguing exercise but before full recovery of force $[1,4,6]$. The trend for an increase in corticomotor excitability in the MS group relative to controls may be a reflection of a greater demand on this central reserve in MS.

There was a comparable increase in corticomotor excitability for both lower limbs with unilateral exercise in controls, whereas in MS there was an increase only for the exercised limb. Previous studies in the upper-limb of healthy subjects have shown that MEP amplitude can be increased in muscles homologous to the exercised muscle [2, 4, 17], although this is not always the case [11]. For the lower limb, there may be additional factors relating to the degree of lateralisation of motor control, through crossed and uncrossed pathways or mediated via the corpus callosum, that could contribute to a bilateral increase in MEP amplitude. The corpus callosum is known to be a target for demyelination in MS [3], and the more lateralised increase in corticomotor excitability in MS may have been a consequence of impaired transcallosal conduction [9, 12]. Alternatively there may have been a greater targeting of corticomotor output to the exercising limb, perhaps due to an increased challenge in performing the task. This would be consistent with the greater increase in MEP amplitude for MS subjects compared to controls. 
To conclude, the present findings provide evidence that mild to moderately affected MS subjects can perform a fatiguing exercise requiring a high level of central motor control, and that this is associated with an increase in corticomotor excitability. Central adaptive motor control mechanisms are likely to enable performance of this task in the presence of force loss and conduction delay, and the increase in corticomotor excitability may reflect a greater demand on these mechanisms in MS. Neural plasticity is likely to be an important factor in maintaining motor performance during fatiguing exercise in MS. 


\section{FIGURE LEGENDS}

\section{Figure 1.}

Change in tap rate during the first and last 5 seconds of each of the 5 bouts of exercise in MS subjects (A) and control subjects (B). Tap rate has been normalised to the first bout of exercise. Dotted lines show the linear regression. Tap rate in the first 5 seconds of each bout increases linearly across bouts for both groups, whereas there is no change in tap rate for the last 5 second period of each bout of exercise.

\section{Figure 2.}

MEP amplitude for the exercised and non-exercised limbs during and after exercise in MS subjects (A) and controls (B). MEP amplitude has been normalised to pre-exercise baseline, and plotted separately for the 4 inter-bout periods, and the 5 post-exercise time points (1 minute intervals). MEP amplitude is increased for the exercised limb during the inter-bout periods for MS and control subjects, and also for the non-exercised limb in controls. 
Table 1: Subject details, Expanded Disability Status Score (EDSS) and walk time.

\begin{tabular}{c|cccccc}
\hline Subject & Age & Sex & $\begin{array}{c}\text { Years Since } \\
\text { Diagnosis }\end{array}$ & $\beta$-interferon & EDSS & Timed walk \\
\hline 1 & \multicolumn{7}{|c}{} & & & & & (sec) \\
\hline 2 & 50 & M & 2.5 & yes & 3.5 & 42 \\
3 & 49 & F & 2 & yes & 3.0 & 41 \\
4 & 33 & M & 4 & yes & 1.0 & 33 \\
5 & 29 & F & 13 & yes & 4.0 & 43 \\
6 & 43 & F & 4 & yes & 2.0 & 43 \\
7 & 43 & F & 13 & yes & 1.0 & 40 \\
8 & 33 & F & 4 & yes & 1.5 & 33 \\
9 & 40 & M & 9 & yes & 0.5 & 29 \\
10 & 64 & F & 26 & no & 2.5 & 48 \\
& & & & & &
\end{tabular}




\section{REFERENCES}

1. Benwell NM, Mastaglia FL, Thickbroom GW (2006) Reduced functional activation after fatiguing exercise is not confined to primary motor areas. Experimental Brain Research $175: 575-583$

2. Bonato C, Zanette G, Manganotti P, Tinazzi M, Bongiovanni G, Polo A, Fiaschi A (1996) 'Direct' and 'crossed' modulation of human motor cortex excitability following exercise. Neuroscience Letters. 216:97-100

3. Gean-Marton AD, Vezina LG, Marton KI, Stimac GK, Peyster RG, Taveras JM, Davis KR (1991) Abnormal corpus callosum: a sensitive and specific indicator of multiple sclerosis. Radiology. 180:215-221

4. Humphry AT, Lloyd-Davies EJ, Teare RJ, Williams KE, Strutton PH, Davey NJ (2004) Specificity and functional impact of post-exercise depression of cortically evoked motor potentials in man. European Journal of Applied Physiology. 92:211-218

5. Kent-Braun JA, Sharma KR, Weiner MW, Miller RG (1994) Effects of exercise on muscle activation and metabolism in multiple sclerosis. Muscle \& Nerve 17:1162-1169

6. Lazarski JP, Ridding MC, Miles TS (2002) Dexterity is not affected by fatigue-induced depression of human motor cortex excitability. Neuroscience Letters. 321:69-72

7. Nielsen JF, Norgaard P (2002) Increased post-exercise facilitation of motor evoked potentials in multiple sclerosis. Clin Neurophysiol 113:1295-1300

8. Perretti A, Balbi P, Orefice G, Trojano L, Marcantonio L, Brescia-Morra V, Ascione S, Manganelli F, Conte G, Santoro L (2004) Post-exercise facilitation and depression of motor evoked potentials to transcranial magnetic stimulation: a study in multiple sclerosis. Clin Neurophysiol 115:2128-2133

9. Petajan JH, White AT (2000) Motor-evoked potentials in response to fatiguing grip 
exercise in multiple sclerosis patients. Clinical Neurophysiology. 111:2188-2195

10. Polman CH, Reingold SC, Edan G, Filippi M, Hartung HP, Kappos L, Lublin FD, Metz LM, McFarland HF, O'Connor PW, Sandberg-Wollheim M, Thompson AJ, Weinshenker BG, Wolinsky JS (2005) Diagnostic criteria for multiple sclerosis: 2005 revisions to the "McDonald Criteria". Annals of Neurology 58:840-846

11. Samii A, Canos M, Ikoma K, Wassermann EM, Hallett M (1997) Absence of facilitation or depression of motor evoked potentials after contralateral homologous muscle activation. Electroencephalography \& Clinical Neurophysiology. 105:241-245

12. Schmierer K, Irlbacher K, Grosse P, Roricht S, Meyer B-U (2002) Correlates of disability in multiple sclerosis detected by transcranial magnetic stimulation. Neurology 59:12181224

13. Schubert M, Wohlfarth K, Rollnik JD, Dengler R (1998) Walking and fatigue in multiple sclerosis: the role of the corticospinal system. Muscle \& Nerve. 21:1068-1070

14. Sheean GL, Murray NM, Rothwell JC, Miller DH, Thompson AJ (1997) An electrophysiological study of the mechanism of fatigue in multiple sclerosis. Brain 120 ( Pt 2):299-315

15. Thickbroom GW, Byrnes ML, Archer SA, Kermode A, F.L. M (2005) Corticomotor organisation and motor function in multiple sclerosis. Journal of Neurology 252:765-771

16. Thickbroom GW, Sacco P, Kermode AG, Archer SA, Byrnes ML, Guilfoyle A, Mastaglia FL (2006) Central motor drive and perception of effort during fatigue in multiple sclerosis. Journal of Neurology 253:1048-1053

17. Zijdewind I, Kernell D (2001) Bilateral interactions during contractions of intrinsic hand muscles. Journal of Neurophysiology. 85:1907-1913 